bility of the diet, especially on those days on which much corn meal was used. With the diet thus supplemented, it was found that a week's daily use of enough of the corn products to furnish over one third of the calories caused no loss of appetite, nor disturbance nor discomfort of digestion, and was entirely satisfactory to the two women and to the man of the family.

Granted that corn products may be substituted for those of wheat to a large extent, or entirely, if desired, without detriment to the attractiveness and palatability of the diet or to the nutritional condition as studied in human subjects by determination of intalie. and output in quantitative experiments of as much as a month's duration, the question may still arise in some minds as to whether long continued use of corn would give results in every way as favorable as the corresponding use of wheat.

The investigations of McCollum, in which laboratory animals have been kept on restricted diets, often for a lifetime, and in several cases for more than one generation, seem well calculated to bring to light any differences in the more obscure factors of food value or in the general wholcsomeness of the two grains, if any such differences exist. In recent summaries of the results of an extended experience with such experiments, McCollum has repeatedly stated that wheat and maize are very similar in their dietary properties. If this seens surprising in view of the well known inadequacy of zein when fed as the sole protein of the diet, it should be recalled that Osborne and Mendel, to whom our lnowledge of the nature of this deficiency of zein is so largely due, have demonstrated also that the other important protein of corn, maize glutelin, is adequate to meet all protein requirements and maintain a normal rate of growth when fed as the sole protein of the diet. They have also shown that zein, while inadequate alone, may yet take the major part in meeting the protein requirements, either of maintenance or of growth, when it is supplemented by a much smaller amount of milk protein.

Persistent use of a diet consisting too exclusively of corn products may lead to unfavorable results, but this is true of the other grains as well: To quote a very recent article by McCollum: "When an animal is fed solely on a single kind of seed, regardless of the kind of plant from which it is derived, loss of weight ensues and death is certain to supervene." This is as true of wheat as of corn, and is no argument against the partial or complete substitution of the one for the other. To the slight extent that two such similar seeds may supplement each other, the substitution of corn for, say, half the wheat ordinarily eaten may improve the biologic value of a diet drawn largely from these grains. Seeds should, however, be supplemented not so much by other seeds as by leaves and tubers, and still better by milk. Quoting McCollum again: "Moderate amounts of milk supplement the deficiencies of the seeds most satisfactorily, and its use forms the greatest factor of safety in our diet."

It is probably no longer necessary to point out that, if pellagra is due to faulty diet, the fault lies not in the use of corn, but in the fact that the diet contains too little of foods of other types. Lusk ${ }^{4}$ has recently stated the case of pellagra in the corn belt and the use of corn as food as follows:

It must be here definitely stated that the use of corn meal is not the cause of pellagra, provided the right kind of other foods be taken with it. . . . Pellagra is generally believed

4. Lusk, Graham: Scient. Month., 1917, 5, 298-309. to be produced by a too exclusive use of highly milled corn and wheat flour in association with salt meats and canned goods, all of which are deficient in vitamins. The arlministration of fresh milk is naturally indicated. Goldberger states that after the addition of milk to the diet of a pellagrin, the typical clinical picture of pellagra no longer persists. The poor in the mill towns of the South lived too exclusively on a corn diet without admixture of milk or fresh animal food or even of cabbage. and pellagra has been the consequence.

The Food Administrator asks us to eat corn bread and save the wheat for export. It is a very small sacrifice to eat corn bread at one meal or more a day. Indian corn saved our New England ancestors from starvation, and we can in part sulsstitute it for our wheat and send the latter abroad to spare others from starvation. The simplest elements of patriotism demand that we do this.

The plea for a fuller use of corn as human food in place of a part of the wheat which we ordinarily consume gains new emphasis and significance from the fact that the wheat situation is becoming constantly more acute, and all indications are that even with a good crop this summer, economy of wheat will continue to be a pressing problem for at least the coming year, whatever may be the fortunes of war.

\section{SKIN GRAFTING*}

\section{J. C. MASSON, M.D.} ROCHESTER, MINN.

At no time in the world's history have there been under treatment so many persons injured and cripn!ed as now. As a great majority of these are suffering from infected wounds, frequently with a great deal of sloughing and destruction of extensive areas of skin and superficial tissues, anything that will tend to hasten healing and cover raw surfaces will be a great benefit to the patient, and a great saving in time and money.

Much has been done in the way of simplifying dressings, and in the control of infection by the scientific application of time-honored surgical principles, and the introduction of antiseptics that have a maximum bactericidal action and a minimum destructive action on the tissues. I shall not, however, dwell on the treatment of wounds or on the major surgical operations indicated, but shall confine myself to the final stage of the treatment of most infected wounds, that is the covering of the raw surfaces with skin.

A great many wounds primarily infected are, under modern methods, made rapidly sterile, as shown by the disappearance of pus and bacteria, and may then be closed with impunity like any fresh wound. But in many of these cases, when there has been extensive destruction of skin, the healthy edges of the raw surfaces cannot be brought together by the ordinary plastic method, and skin grafting must be resorted to.

In reviewing the history of surgery it is not surprising to note that skin grafting was one of the first operations attempted; in fact, it is asserted that the ancient Hindus and Egyptians successfully transplanted skin before the Christian era, and it is generally accepted that this operation has been done with varying degrees of success in different parts of the world ever since. It was not, however, until 1869, following the reading of a paper and the presentation of a case by Reverdin before the Société de chirurgie

* From the Mayo Clinic.

* Presented before the Olmsted County Medical Association, Rochester, Minn., April 10, 1918. 
in Paris, that it was accepted as a regular surgical procedure, and since that time a great deal has been written on the subject.

The grafts, as first suggested by Reverdin, were very similar to those that we now accept as probably

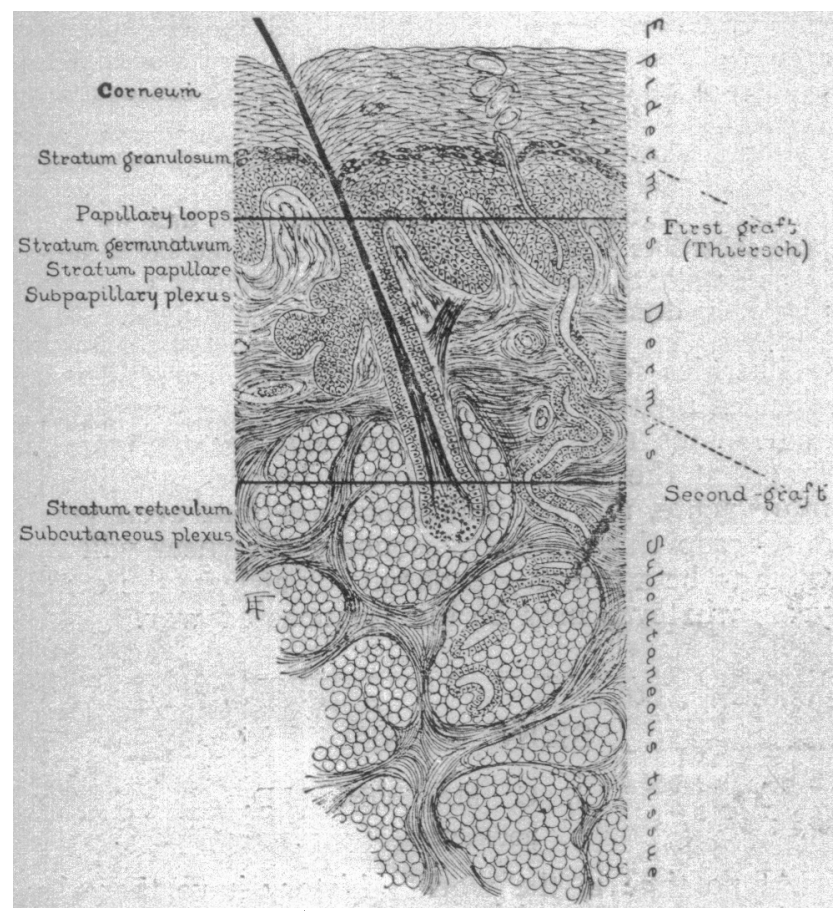

Fig. 1.-Normal skin, showing part utilized in Thiersch graft, and the deep part utilized by a new method.

of the best type of graft for use in the ordinary case in which closure of the wound is the all important consideration. These are small island grafts, principally epidermic, but containing some dermis in the center, and called by Davis small, deep skin grafts. The other accepted and useful grafts are known as Thiersch grafts and Wolfe grafts. The former are thin sheets of skin, removed with a razor, and include all the layers of the epidermis and possibly a portion of the dermis; the latter are composed of the entire thickness of the skin, and when successfully used are the most satisfactory.

Skin grafting is divided into three distinct types, depending entirely on where the grafts are obtained: (1) autoplastic grafts-skin taken from another part of the body of the patient; (2) isoplastic grafts-skin taken from another person, and (3) zooplastic grafts -skin taken from one of the lower animals. All authorities agree that the autograft is the most satisfactory, and a great many hesitate to use even isografts when it is possible to get skin from the patient.

I myself have had no experience in the use of zoodermic grafts, but am satisfied that there is a much larger field of usefulness for the isograft than is generally believed. When one takes into consideration the ease with which good grafting material may be obtained in the operating room without discomfort or inconvenience to the donor, and with the removal of all dread of the operation from the recipient, one is convinced that it should certainly be more frequently practiced. In isografting, the cause of failure should not always be attributed to the graft itself, since it may be due to the patient, to the condition of the wound or to the after-care and dressing, et: This problem is especially difficult to solve in p:ivate prac- tice, in which time is such an important consideration to the patient and little or no help can be obtained by experimental work.

In all the patients requiring skin grafting who were under my care during the past year, the blood of the donor, as well as that of the recipient, has been tested for agglutination. The results have been very interesting and instructive, and I feel sure will add a great deal to the popularity of the use of the isograft. In many cases the method will obviate the unnecessary

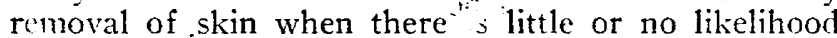
of its taking. I have tested the princiole with the three varieties of grafts, and am satisfied that blood grouning is just as important for good results in skin grafting as it is necessary in transfusion, and that it is governed by the same principles. ${ }^{1}$ While the results obtained are not positive, nevertheless, I have never had the skin take which was removed from a donor whose red blood corpuscles were agglutinated by the serum of the patient. The results in all other cases have been very satisfactory, almost, if not entirely, equal to autodermic grafting. In most cases in which a donor's skin is to be used, a Wassermann test should be made to guard against syphilitic infection.

In any case in which it is considered expedient to resort to skin graftirg, the first consideration is to have the raw suface in a healthy condition. Experience shows that only poor results can be expected on an indolent ulcer, one covered with exuberant granulation tissue or one in which the microscopic slide shows numerous bacteria. Grafting may be satisfactorily done on fresh raw surfaces, but pedicle or plastic grafts are, as a rule, more satisfactory in these cases,

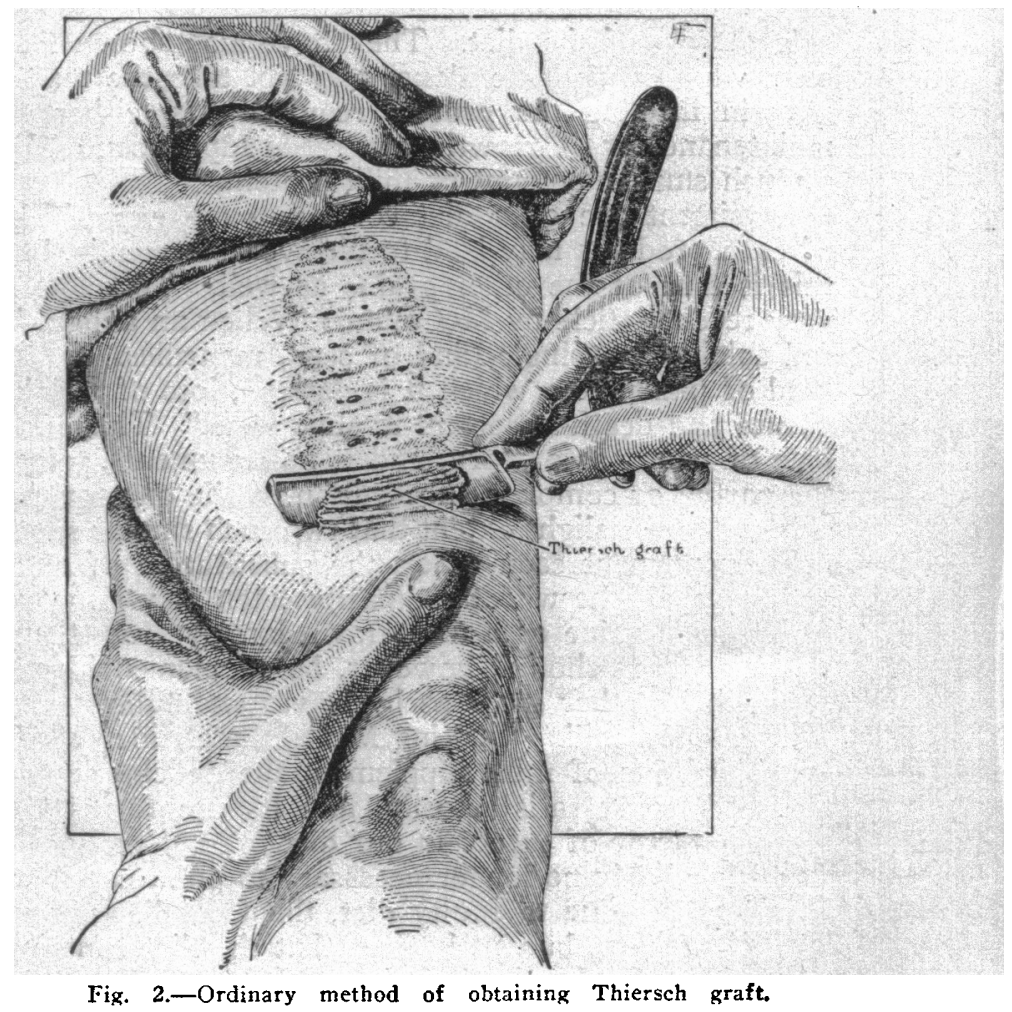

and true skin grafting is much more frequently indicated as a secondary method in closing open wounds or ulcers.

1. Sanford, A. H.: Selection of the Donor for Transfusion, JournilLancet, 1917, $\mathbf{8 7}, 698$. 
In all chronic ulcers, especially those resulting from roentgen-ray or electric burns, the first consideration is to improve the circulation in the part, and to stimulate healing by the removal of the unhealthy granulations, either by curetting or by excision of the ulcer. Grafts may be placed immediately, but in most cases it is advisable to apply to the raw surface hot saline or boracic dressings until it is covered with healthy granulations, and in some cases it is desirable to use scarlet red ointment ( 8 r cent.) once in two days until a healthy condition of the granulating surface is obtained and a small pellicle of new skin appears along the edge. Satisfactory results can then be expected from grafting.

In the more recent cases, such as are so often seen in patients following burns, extensive superficial infections, or radical operations in malignancy, the best plan is to apply hot saline, neutral solution of chlorinated soda (Dakin's solution) or dichloramin- $T$, until the wound is made sterile, as shown by smears on three consecutive days. The grafts may then be applied direct,

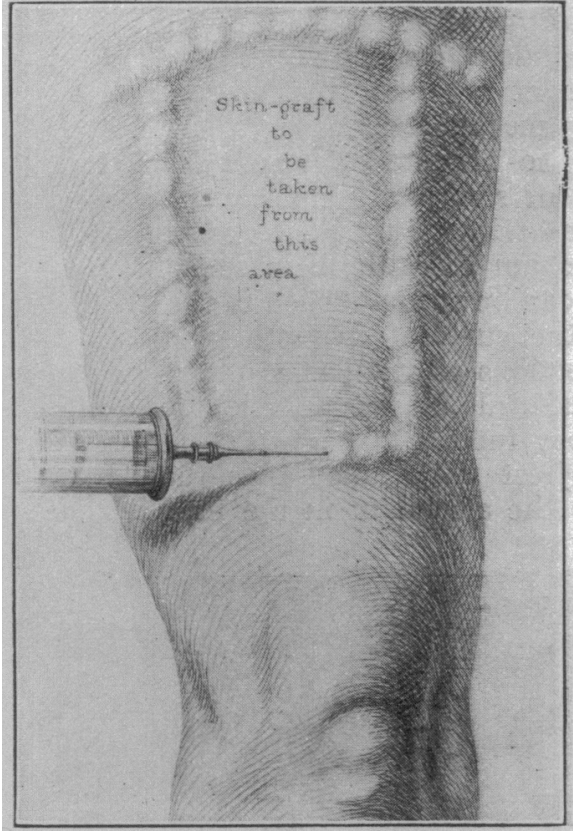

Fig. 3.-Superficial blocking with local anesthesia for skin grafting.

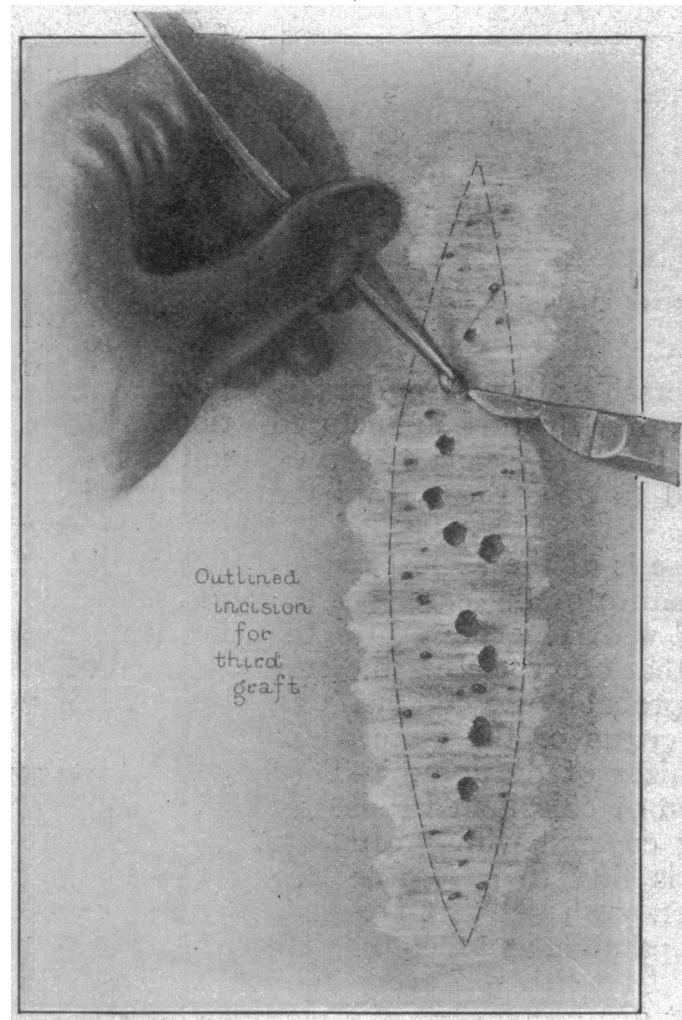

Fig. 4.-Further utilization of area from which Thiersch graft has been removed
In deciding where to obtain grafts, the type of skin desired should naturally be considered. For most cases the skin from the anterior and outer surface of the thigh or upper arm will be found best, whether it be from the patient himself or from a donor.

General anesthesia is frequently advisable, but even extensive grafts may be obtained under local anesthesia with very little discomfort to the donor, although so far as the operation or the surgeon is concerned, the latter has no advantage. It is much easier, however, to obtain a donor if it is understood that he will not be anesthetized.

It makes very little difference what method is used in preparing the skin, and the iodin method is as satisfactory as any. The area is first cleaned with benzin containing $1: 1,000$ iodin, and after drying, it is given two coats of 3.5 per cent. iodin in alcohol. Figure 1 is a transverse section of the normal skin. A Thiersch or, if the granulations are exuberant, they may first be curetted away or rubbed off with a piece of dry gauze, controlling hemorrhage by pressure with gauze wrung dry from hot saline solution and applying the grafts immediately, pressing them well into place with moist gauze. If there is much oozing it is better to delay the placing of grafts for twenty-four hours, keeping up the hot moist dressings.

The types of grafts to be used and where they shall be obtained are very important considerations. The full thickness, or Wolfe graft, gives the most normal looking and best functionating skin, but has the disadvantage that it is not so apt to take as is either the Reverdin or Thiersch graft. However, in annular ulcers of the extremities, around joints, or in dealing with extensive areas, it is necessary to have at least a part of the surface covered with grafts of the entire thickness of tha skin. graft is then cut after the ordinary method (Fig. 2) If local anesthesia is to be used, the skin is injected, as shown in Figure 3. If the skin is thick, a second layer may be removed from the same area in the same way, or small island grafts may be taken from the center of the raw surface to include some of the deeper layers of the epidermis and some of the superficial layers of the dermis (Fig. 4).

It is advisable to reduce the size of the wound, especially if much of the dermis has been removed, by cutting an elliptical piece of tissue (Fig. 5) and suturing the edges together with silkworm gut and horsehair. The tissue thus removed may also be utilized for grafting by cutting it into small sectional grafts (a term used by Colebrook and Fleming) and applying just as the Reverdin grafts are applied. Figure 6 shows the wound covered with Thiersch grafts and small, deep grafts taken from the denuded 
area. The advantages of this method are: (1) Grafting material equal to twice the denuded area is obtained; (2) it is easily and quickly done and is especially suited for local anesthesia, and (3) the clean cut wound may be expected to heal by primary union after being sutured with silkworm gut and horsehair, which has a distinct advantage over the raw area left after the Thiersch method or the necessary multiple wounds of the Reverdin method.

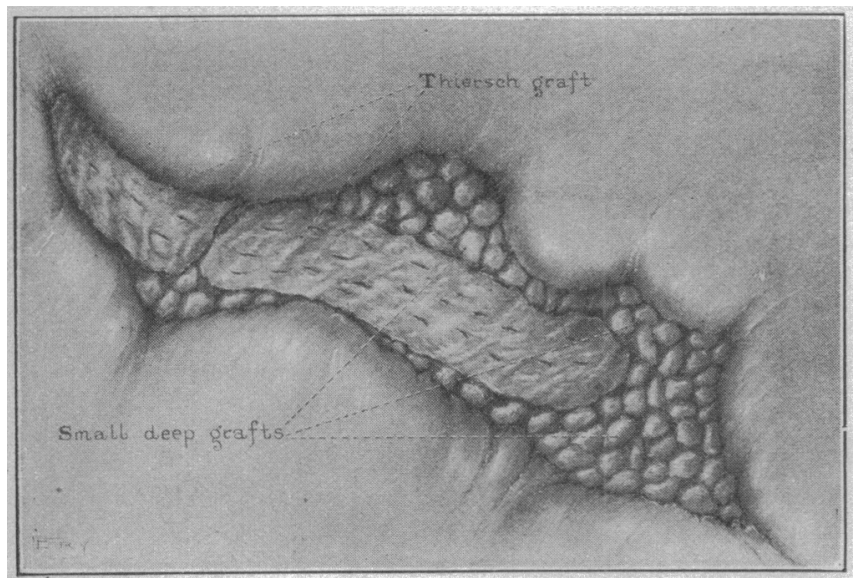

Fig. 6.-Wound covered with Thiersch grafts and small deep grafts taken from denuded area.

If large grafts, either Thiersch or those from superficial dermic layers, are used, they should be punctured at numerous points to allow the free escape of serum, which would otherwise tend to float them from the surface.

The type of dressing to use is of the utmost importance and varies with the nature of the case. If the wound is completely covered with Thiersch grafts, the open method of treatment, that of protecting the surface with a wire screen, is probably the best, with an occasional removal of any crusts or thick secretions and the application by atomizer of dichloramin-T ( 4 per cent.) or neutral solution of chlorinated soda. If the wound is only partially covered with grafts, the most satisfactory dressing is first to cover the raw surface and grafts with open-mesh net that has been previously impregnated with paraffin, and then to apply a wet dressing, which should be changed every four hours for three days without disturbing the paraffined net, the latter being held in place by sutures or by applying soft paraffin along the edges to fix it to the surrounding skin. Open air treatment is then combined with hot dressings, the hot dressings being used at night and the wound left exposed during the greater part of the day and sprayed once or twice during the twelve hours with neutral solution of chlorinated soda. As a rule, grafts have taken well in one week's time, after which sterile petrolatum makes a very satisfactory dressing.

Frequently the paraffined net becomes firmly adherent to the grafts while wet dressings are being combined with open air treatment; but it may easily be freed by applying a liberal petrolatum dressing or by spraying with liquid petrolatum for from four to six hours before attempting its removal.

Body Heat and Oxidation.-The total quantity of heat produced by the body is a measure of the intensity of the oxidation of carbohydrate, fat, and protein within the body.-Lusk, Food in War Time.

\section{PHYSIOLOGIC COLECTOMY IN CHRONIC TOXEMIAS OF INTESTINAL ORIGIN}

\section{CHARLES A. L. REED, M.D.} CINCINNATI

The cumulative experience of both physicians and surgeons engaged in a large way with the treatment of gastro-intestinal conditions, and of experimental scientists in dealing with these problems, tends to confirm what may be expressed as at least tentative conclusions as follows:

1. The origin of certain toxins and of the now recognized anaphylatoxin ${ }^{1}$ demonstrated to be present in certain toxemias, both convulsive and nonconvulsive in type, is due to certain conditions of stasis in the gastro-intestinal tract.

2. Stasis in the gastro-intestinal tract implies (a) fecal retardation in either the small intestine or the colon or both; (b) venous retardation in either the mesocolic circulation or the mesenteric circulation or both, and $(c)$ retardation in the lymphatic circulation of both the mesocolon and the mesentery.

3. These three forms of stasis are associated with and obviously dependent on some form of gastrointestinal dispiacement or other distortion, and are, therefore, primarily mechanical in origin.

4. There can be no permanent benefit from treatment in these cases of stasis with consequent constitutional manifestations without first a correction of the initial link in the chain of pathologic sequences just enumerated. This can be accomplished in the majority of cases only by the surgical correction of the displacements or distortions of the gastro-intestinal tract as revealed by careful diagnostic methods, often including exploratory incision.

It is of the highest importance that the general practitioner who is the attendant in the first instance

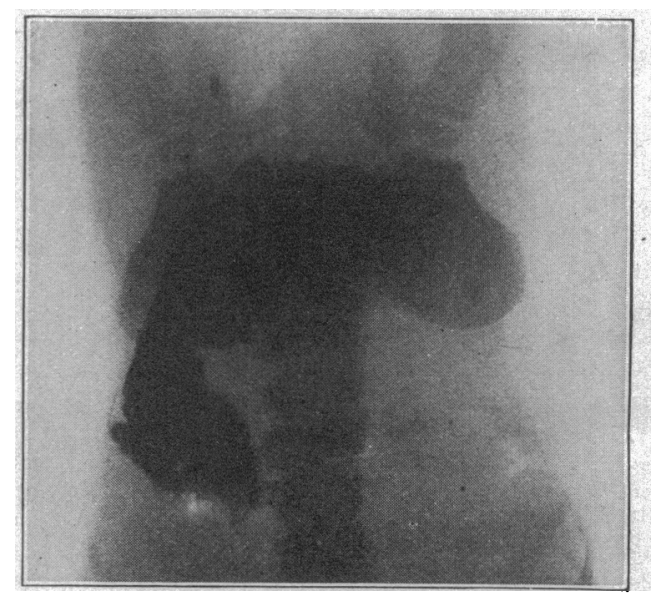

Fig. 1.-Necessity for physiologic colectomy (colonic exclusion), in a child aged $31 / 2$ years. Colon stasis with chronic colitis. Ileac stasis due to adhesions at terminal ileum. Resulting chronic convulsive toxemia. Fifty-six seizures in week preceding operation; no seizure to date (eleven months) since operation. (Internal conditions shown
in Fig. 2.)

of these unfortunate persons should know just what surgical measures are available for their relief, and just what results may reasonably be expected. In this connection it is important at the very outset to emphasize the rule that either no surgery at all or the least

1. Novy, F. G., and DeKruiff, P. H.: Anaphylatoxin and Anaphylaxis, The Journal A. M. A., May 26, 1917, p. 1524. 